\title{
Integration and representation in rats' serial pattern learning in the T-maze
}

\author{
J. S. COHEN, A. SIMPSON, K. WESTLAKE, and P. HAMELIN \\ University of Windsor, Windsor, Ontario, Canada
}

\begin{abstract}
Rats were exposed to three-trial series consisting of reinforced $(\mathrm{R})$ trials and one nonreinforced $(\mathrm{N})$ trial in a fixed order, RRN and RNR (Experiments 1 and 2) or NRR and RRN (Experiment 3), on extended visually distinct runways in a T-maze. When initially presented with the same sequence on each series in a session (separate presentations) with the same runway on all trials within a series (Experiments 1 and 3), all the rats developed slower running speeds on $\mathrm{N}$ than on $\mathrm{R}$ trials. When a runway was sometimes changed between the first and next two trials during separate presentations training (Experiment 2) or both sequences were later intermixed within each session in each experiment, only rats exposed to each sequence on a specific runway maintained these serial running patterns. Rats displayed serial running patterns on a test RNN sequence similar to that on the RNR sequence (Experiment 2), as would be predicted by an intertrial association model of serial pattern learning (Capaldi \& Molina, 1979), but responded on test RRR and NRN sequences (Experiment 3) as would be predicted by an ordinal-trialtag/intratrial association model (Burns, Wiley, \& Payne, 1986). Results from test series of free-choice trials in Experiments 1 and 2 failed to support a prediction of the intratrial association model that these rats would integrate RRN and RNR sequences. Rather than always selecting a baited runway on both the second and the third free-choice trials, the rats only selected a baited runway on the third trial on the basis of their choice on the second trial, as would be predicted by the intertrial association model. Only after experiencing all possible outcome sequences during forced-choice training in Experiment 3 did these rats predominantly select a baited runway on every free-choice trial.
\end{abstract}

When repeatedly exposed to a fixed sequence of rewarded $(\mathrm{R})$ and nonrewarded $(\mathrm{N})$ trials in a runway, rats typically learn to run more slowly on the nonbaited trials (Capaldi \& Verry, 1981; Capaldi, Verry, Nawrocki, \& Miller, 1984; Hulse, 1978). Separating each series of trials by long intervals or by alternating them between visually distinct runways facilitates such serial pattern learning (Fountain, Henne, \& Hulse, 1984). This effect may be analogous to how chunking verbal and numerical items in lists with phrasing or grouping cues improves human recall performance (Bower \& Springston, 1970; Bower \& Winzenz, 1969). It is noteworthy that rats can acquire different serial patterns from different sequences when each is separately presented over sessions (e.g., $\mathrm{RN}$ and $\mathrm{RRN}$; Capaldi, Miller, Alptekin, \& Barry, 1990) or always occurs in a fixed order within each session (e.g., RNN and RNR; Capaldi, Nawrocki, \& Verry, 1983). According to the hierarchical model of serial pattern chunking (Capaldi,

The present research was supported by a grant to the first author from the Natural Sciences and Engineering Council of Canada and by funds to the second and third authors from the Ontario Undergraduate Work-Study program administered by the University of Windsor. The first and second experiments served as undergraduate B.A. and B.Sc. theses for the second and fourth authors, respectively. Portions of this study were presented at the combined Canadian Brain, Behaviour, and Cognitive Sciences Society/Experimental Psychology Society meetings at Cambridge University, (July 2000). Correspondence concerning this article should be addressed to J. S. Cohen, Department of Psychology, University of Windsor, Windsor, ON, N9B 3P4 Canada (e-mail: jcohen@uwindsor.ca).
1992), these effects indicate that rats can combine or integrate different series chunks into higher level list chunks.

In research on the hierarchical chunking model in our laboratory (Cohen, Westlake, \& Pepin, 2001), we investigated whether rats could combine two sequences, RRN and RNR, into an even higher level, RRR, super chunk. In that study, some rats experienced each sequence on specifically distinct runways, left black and right striped runways in a T-maze (associated-runways group), whereas other rats received each sequence equally on each runway (nonassociated-runways group). Each three-trial series was separated by a relatively long 60 -sec delay in a darkened start chamber, the phrasing cue, whereas each trial within the series was separated by a very short 10 -sec delay in the lit start chamber. Although both groups developed slower speeds on the $\mathrm{N}$ trial when each sequence was presented on separate sessions, only the associated-runways group maintained this serial running pattern when sequences were later intermixed within sessions. The rats in the nonassociated-runways group continued to run slower on the last trial within the RRN sequence during intermixed presentations, however, suggesting that they had learned that the outcome on the second trial of a series signaled the opposite outcome on the third trial.

The rats in the associated-runways group were expected to integrate the RRN and RNR list chunks into an RRR super chunk if they had learned which runway was baited on each trial within a series. For example, if it has always been exposed to the RRN sequence on the left black run- 
way and the RNR sequence on the right striped runway, a rat may have learned that either runway will be baited on the first trial of any series but only the left runway will be baited on the second trial and only the right runway will be baited on the third trial. Thus, if allowed to choose between runways on each trial following acquisition of each list chunk on forced-choice trials, it should select the left, baited runway on the second trial and the right, baited runway on the third trial. This prediction is based on the ordinal trial "position" model of serial learning (Burns, Dunkman, \& Detloff, 1999; Burns, Wiley, \& Payne, 1986), which maintains that rats learn a fixed sequence of different outcomes by associating each with a trial's temporal tag (1st, 2nd, 3rd position) within the series-that is, by forming intratrial associations between each tagged trial and its outcome. In our preparation, rats in the associated-runways group would form the following intratrial associations: that either runway is baited on a trial ordinally tagged as first in a series but that a specific runway is baited on trials ordinally tagged as second and third in a series.

The rats in the nonassociated-runways group, on the other hand, could not know which runway would be baited on the second free-choice trial but might be able to select the baited runway on the last free-choice trial by remembering the specific runway chosen on the second trial and whether it had been baited. This reasoning corresponds to the intertrial-association/sequential-memory model of serial learning (Capaldi \& Molina, 1979). According to this model, rats accurately anticipate a current trial's outcome by retrieving the memory of previous associated events, such as delays, runways, and the outcomes of previous trials. Thus, so-called phrasing cues serve simply as events that signal (become associated with) a specific outcome on the next trial of a series. In Cohen, Westlake, and Pepin (2001), for example, rats would learn that a rewarded trial will always occur after a long delay but that an intermittently, unpredictably rewarded trial will always follow a short delay and will predict the opposite outcome for the next trial after another short delay in the lit start box. Furthermore, the type of runway following upon a long delay will also become associated with or predict the next runway for all the rats, as well as the next trial's outcome for the rats in the associated-runways group. Consequently, no specific ordinal tag for each trial is needed for higher order list chunking and maintenance of serial pattern responding on intermixed sequences in the associated-runways group. On the other hand, this model has greater difficulty in predicting the integration of both serial patterns into a super RRR chunk.

When free-choice test series were carried out, however, neither group of animals seemed to use either underlying type of association or representation for forming or integrating the two sequences. Although the two groups did differ in their free-choice patterns on their first two trials, both selected a baited arm on one (the first) trial or on all trials about $25 \%$ of the time and on two trials about $50 \%$ of the time, as would be expected by chance. Two reasons may account for the failure to observe either type of inte- gration of each outcome sequence. One possibility is that free-choice testing was too novel a change following extensive forced-choice training for the rats to transfer information from the latter. The other possibility is that free-choice testing may be relatively insensitive in uncovering these type of associations. In either case, forcedchoice tests similar to those used by Burns et al. (1999) might be more sensitive for revealing the underlying associations responsible for serial pattern learning and integration. In their study, rats were initially trained on two sequences, ZNN and XNY, each presented on a distinctly different runway, where $\mathrm{X}, \mathrm{Y}$, and $\mathrm{Z}$ were different food types. Thus, the rats could develop intertrial associations between initial food type and the third trial's outcome or intratrial associations between the outcome of each ordinally tagged trial and its runway. Following initial training, the rats received two types of probe test sequences: one in which type of food on the first trial of a probe test series signaled the opposite sequence from that presented on the specific runway and another in which each runway remained unbaited on all trials. The rats continued to display serial running patterns based only on the specific runway presented on either type of probe series, suggesting that intratrial associations were dominant over intertrial associations.

These considerations prompted a further investigation of whether and how rats might represent and integrate complementary three-trial sequences. The present study consisted of three experiments in which integration and representation based on intratrial (ordinal position) and intertrial (sequential memory) associations were examined with free-choice and forced-choice tests.

\section{EXPERIMENT 1}

The rats in the associated-runways group in Cohen, Westlake, and Pepin (2001, Experiment 2) were more likely to choose the runway associated with the RRN sequence on their first two free-choice trials, but not in their final free-choice trial. The rats in the nonassociatedrunways group, however, alternated their runway choices during free-choice tests. This difference most likely resulted from initial training and testing on forced-choice and free-choice two-trial sequences (RR and RN) in Experiment 1 . This possibility prompted us to conduct a modified version of Experiment 2. In the present experiment, we first familiarized rats with forced- and free-choice three-trial series consisting of an RRR sequence on each runway before exposing them to the RRN and RNR sequences. As in the previous study, half the rats received each sequence paired with a specific, visually distinct runway in the T-maze (associated-runways group), whereas the other half were equally exposed to each sequence on each runway (nonassociated-runways group). In the first of the forced-choice phases, the rats were presented one sequence repeatedly within a session, so that they experienced each sequence equally over sessions (separatedpresentations condition). In the second forced-choice phase, each sequence was equally intermixed within each 
session (intermixed-presentations condition). As in the original experiment, the rats in both groups were expected to reduce their speed on the $\mathrm{N}$ trial within each sequence during the separate-presentationsphase. During the intermixedpresentations phase, the associated-runways group was expected to maintain this serial response pattern within each sequence, but the nonassociated-runways group was expected to do so only within the RRN sequence.

Our major modifications occurred for testing, which included a forced-choice phase after a free-choice phase. During the first test phase, the rats continued to be forced down one runway on the first trial but were allowed to choose either runway on the next two trials within each series in the first half of the free-choice test phase. In the second half, they were allowed to choose either runway on all three trials, as in the original study. We employed the partial free-choice design on the first six sessions to reduce any tendency the rats in the associated-runways group might have to initially choose the runway associated with the RRN sequence on the first free-choice trial in the last six sessions.

The second test phase was based on that used by Burns et al. (1999). It consisted of intermixed presentations of each sequence, as in the second training phase, except that half the series within each sequence consisted of forcing the rats, on the first trial, down the runway opposite from that on the second and third trials (changed-runway condition). On the remaining series, the rats continued to be forced down the same runway on all three trials within a series (same-runway condition). According to the ordinal trial position hypothesis, varying the runway between the first and the next two trials should not affect the associated-runways group's serial response pattern. That is, these rats should continue to run more slowly on the $\mathrm{N}$ trials within either sequence, regardless of which runway they were forced down on the first trial. According to the intertrial, sequential memory hypothesis, changing the runway between the first and the second trials should disrupt these rats' serial pattern performance. More specifically, their speeds on the second trial in each sequence should be moderated by the type of runway presented on the first trial. Thus, on the second trial, these rats would be more likely to run faster and slower within the RNR sequence and RRN sequence, respectively, under the changed-runway condition than under the same-runway condition. The rats in the nonassociated group were not expected to be much affected by this factor, because they could determine the outcome on the third trial only by remembering the outcome on the second trial.

\section{Method}

\section{Subjects}

Twelve male Long-Evans hooded rats from the Charles River Breeding Farms, St. Constant, Quebec, served in this experiment. They were veterans of an earlier simultaneous object discrimination study in a different object-choice apparatus (Cohen, Drummond, \& Terrelonge, 2001), were approximately 150 days old, and weighed over $450 \mathrm{~g}$ at the start of this experiment. The same general maintenance and housing conditions as those in Cohen, Westlake, and Pepin
(2001) remained in effect. Each rat received its daily ration of $20 \mathrm{~g}$ of Purina Rodent Chow for $2 \mathrm{~h}$ following each experimental session, which kept them at approximately $90 \%$ of their free-feeding weight. Water was always available in group and individual holding cages.

\section{Apparatus}

The apparatus in Cohen, Westlake, and Pepin (2001; see their Figure 1) was used. It consisted of an enclosed, black, wooden T-maze with a 7.5 -cm-wide and 160 -cm-long metal runway extending from each side arm. A return alley, located $20 \mathrm{~cm}$ from the beginning of each runway, curved back to a standard stainless steel holding cage that served as a start chamber attached to the center arm. The interior of the left runway was painted flat black, whereas that of the right runway contained black and white stripes on the walls and the floor from the return alley to the goal area. A movable food tray containing eight food wells could be slid in and out of end of each runway. The maze was illuminated by a $40-\mathrm{W}$ incandescent light over each runway and behind a translucent plastic panel at the end of the center arm.

\section{Procedure}

Familiarization training. Procedures for shaping rats to run down each runway for four sunflower seeds and to return to the start chamber were the same as those in Cohen, Westlake, and Pepin (2001). The experimenter began a session by placing the rat in the start chamber in the darkened maze for $60 \mathrm{sec}$ before illuminating the maze and lifting the center arm door for the first trial. As soon as a rat in the start chamber faced the center arm of the T-maze, the experimenter lifted the start chamber's clear guillotine door to the center alley of the T-maze. This activated a silent digital clock that stopped when the rat crossed a photo-beam, $190 \mathrm{~cm}$ from the start chamber and $10 \mathrm{~cm}$ from the end of either runway (its respective goal area). The experimenter lowered the start chamber's guillotine door when the rat entered the center arm and lowered the side arm's guillotine door when it entered the side runway. A food tray, baited with four shelled unsalted roasted sunflower seeds, remained outside the end of each runway until the rat broke the beam, whereupon the experimenter either slid it into the goal area or removed it from its location outside the runway, depending on the trial's programmed outcome. The experimenter then slid open the door to the start chamber from the return alley and closed it when the rat reentered the start chamber. Throughout the experiment, the maze remained illuminated while the rat waited in the start chamber for approximately $10 \mathrm{sec}$ between trials within a series but was darkened for $60 \mathrm{sec}$ (the phrasing cue) while it waited in the start chamber between series. The experimenter rebaited the food trays during these intertrial and interseries intervals. The experimenter removed the rat from the apparatus after it returned to the start chamber following the last trial in a session. The experimenter recorded each rat's running time (to the nearest $0.1 \mathrm{sec}$ ) from when the start chamber door to the center arm was raised to when the rat intersected the beam at the end of a runway in front of the goal area. Running times were transformed into centimeters/second speed scores for statistical analysis.

Forced-choice and free-choice RRR sequence training. The rats were trained first on series of a single RRR sequence on each runway for 24 sessions, to acquaint them with the basic forcedchoice and free-choice procedures. Each session contained eight series of three massed trials throughout this experiment. The rats were forced down a baited arm on each trial within a series during the first 12 sessions. Each runway was presented on four series in a random order within each session, without the same runway occurring on more than two series in a row. Over the last 12 sessions, the rats were allowed to choose either baited arm on each trial within each series. The next four phases constituted the actual experiment, as follows.

Phase 1: Separate forced-choice presentations of RRN and RNR sequences. The rats were randomly assigned to two equal groups (associated-r unways group and nonassociated-r unways group) and experienced 48 sessions, half of which contained series 
of the RRN sequence and the other half of which consisted of the RNR sequence. Each type of session occurred in a random order, without the same sequence occurring more than 2 sessions in a row. Within a session, a specific sequence was available only on one runway. Three rats in the associated-runways group experienced the RRN sequence always on the left (black) runway and the RNR sequence always on the right (striped) runway; the other 3 rats received the opposite pairings. Rats in the nonassociated-runways group, however, were exposed to each sequence equally on each runway in a random order. Consequently, each rat received each sequence on 192 series.

Phase 2: Intermixed presentations of RRN and RNR sequences. Following the separate-presentations phase, the rats received each sequence on 4 series within in each session in a random order, without the same sequence occurring on more than 2 series in a row. This phase consisted of 24 sessions, so that each sequence occurred on 96 series. The rats in the associated-runways group continued to experience each sequence on a specific runway, whereas the rats in the nonassociated-runways group continued to be exposed to each sequence equally on each runway in a random order.

Phase 3: Free-choice test. The first of the two test phases consisted of 12 sessions. On the first 6 sessions, the rats were forced to one runway on the first trial but were able to choose between both runways on the next two trials in each series in each session. The available runway on the first trial was randomly determined, so that each occurred equally without being presented on more than two series in a row. Both runways were open on every trial within each series on the final 6 sessions. Whereas both runways were baited on the first trial of each series, only one runway was baited on the next trial, and the opposite runway was baited on the third trial. Which runway was baited on the second and third trials depended on which sequence had been paired with which runway during forced-choice training for the rats in the associated-runways group. For the rats in the nonassociated-runways group, either runway was baited, in an equal and random order, on the second trial, with the opposite runway being baited on the third trial within any series.

Phase 4: Forced-choice test. The rats received a block of 24 sessions of forced-choice series of RRN and RNR sequences intermixed within each session. The rats continued to be forced down the same runway on each trial for half the series within each sequence, as in Phase 2 (same-runway condition). On the other series, however, they were forced down a runway, on the first trial, opposite that on the next two trials (changed-runway condition). Both runway conditions were randomly presented over series, without either being presented on more than two series on a row.

\section{Results and Discussion}

The results from the appropriate analyses of variance (ANOVAs) and post hoc individual comparisons (NewmanKeuls tests) were considered significant at $p<.05$. The results from the forced-choice training Phases 1 and 2 essentially replicate those in Cohen, Westlake, and Pepin (2001, Experiment 2). Data were averaged over the last 24 sessions in Phase 1 and over the 24 sessions of Phase 2 and were analyzed with a group $\times$ sequence $\times$ trial ANOVA for each phase. When each sequence was separately presented over sessions (Phase 1), both groups similarly reduced their speeds on the $\mathbf{N}$ trial within each sequence (associated-runways group, RRN, 72, 63, 59, and RNR, 71, 54, 69; nonassociated-runways group, RRN, 68, $62,57 ; \mathrm{RNR}, 70, \mathbf{6 0}, 64)$ to yield a significant interaction between sequence and trial $[F(2,20)=10.66]$. When sequences were intermixed, however, the associated-runways group continued serial pattern responding on both (RRN,

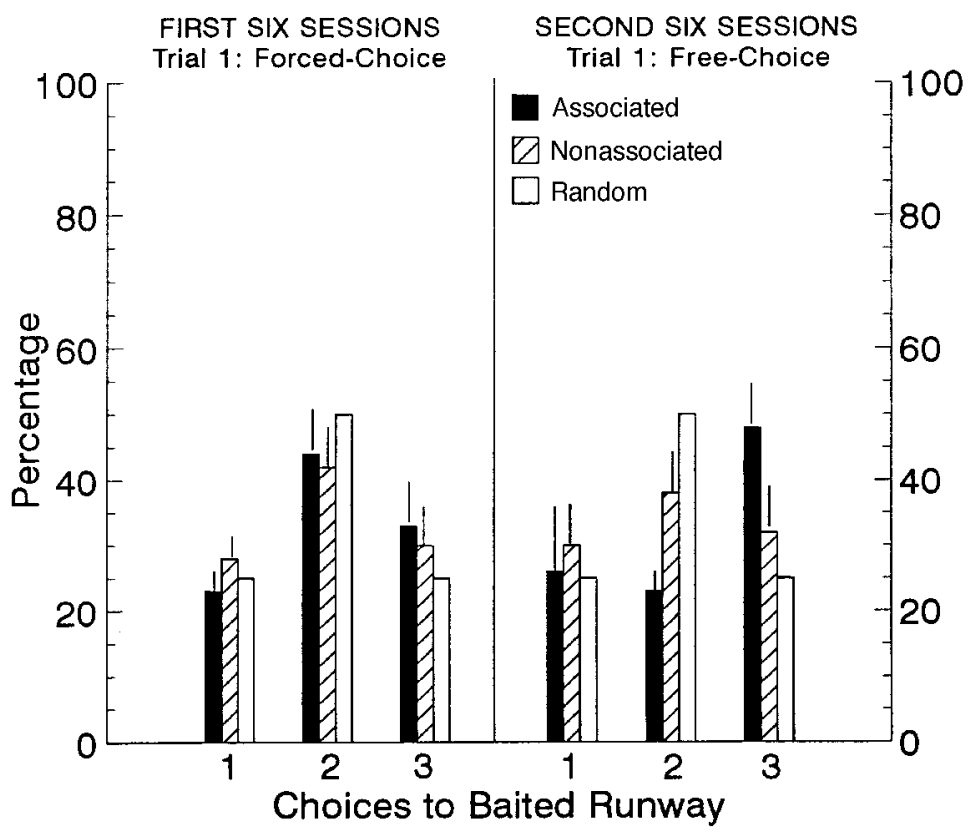

Figure 1. Percentages of choices of a baited runway made on one, two, or all three trials during the free-choice test phase for each group, as compared with that which would occur by random selections in Phase 3 of Experiment 1. Vertical lines at the top of the mean data bar represent $+S E M s$. 
$65,61,49$; RNR, 62, 40, 56), whereas the nonassociatedrunways group did so on the RRN, but not on the RNR sequence (RRN, 66, 64, 57; RNR, 62, 62, 64), to yield a significant interaction among all factors $[F(2,20)=10.01]$.

Phase 3: Free-choice tests. Figure 1 shows the percentage of times the rats in each group chose a baited runway only once, twice, or on all three trials, either when forced to one of the two baited runways on the first trial (first six sessions; left panel) or when allowed to choose between them on every trial (second six sessions; right panel) of each series. These data were compared with percentages that would have occurred had the rats randomly selected runways during free-choice trials (open bars). During the first half of this phase, both groups displayed distributions of choice patterns not significantly different from each other and similar to that expected by chance. A slight but significant effect $[F(1,5)=7.31]$ was found in the associatedrunways group, which selected a baited runway on all trials more often than would be expected by chance. When the rats were able to choose between both runways on all trials in a series over the last six sessions, the groups differed in their distributions of choices. The associated-runways group chose a baited runway on all three free-choice trials not only significantly more often than chance, but also significantly more often than it chose a baited runway on two out of three trials $\left[F_{\mathrm{s}}(1,5)=10.31\right.$ and 11.87], which occurred below chance $[F(1,5)=144.13]$. A similar difference between choosing a baited runway on every trial and choosing it on only the first trial was not significant. The choice patterns of the nonassociated group did not significantly depart from those expected by chance.
The fact that the rats in the associated-runways group developed a clearer preference for the baited runway on every free choice trial only on the last six sessions suggests they were beginning to learn which runway was baited on each free-choice trial. Had we continued these free-choice "test" series, these rats might have eventually learned to always choose the baited runway. Therefore, we cannot conclude that these rats had integrated RRN and RNR sequences into a super RRR sequence chunk based on ordinal trial position associations. Furthermore, the rats in the nonassociated-runways group were unable to use intertrial associations from forced-choice training, since they were unable to always choose the baited runway on the last free-choice trial.

Phase 4: Forced-choice tests. Figure 2 shows the mean speeds on each trial within each sequence on the samerunway (left panel) and changed-runway (right panel) conditions for each group. Speed data were analyzed with a separate group $\times$ sequence $\times$ trial ANOVA for each runway condition. As can be seen in left panel (same-runway condition) and was confirmed by a significant three-way interaction $[F(2,20)=19.09]$, only the associated-runways group maintained its serial pattern responding, within the RNR sequence, by reducing its speed on the second trial and then increasing it on the third. Both groups reduced their speeds on the second trial and reduced them further on the third trial within the RRN sequence. As can be seen in the right panel (changed-runway condition) and was confirmed by a significant three-way interaction $[F(2,20)=$ 5.99], the associated-runways group's serial pattern responding within the RNR sequence was disrupted, as pre-

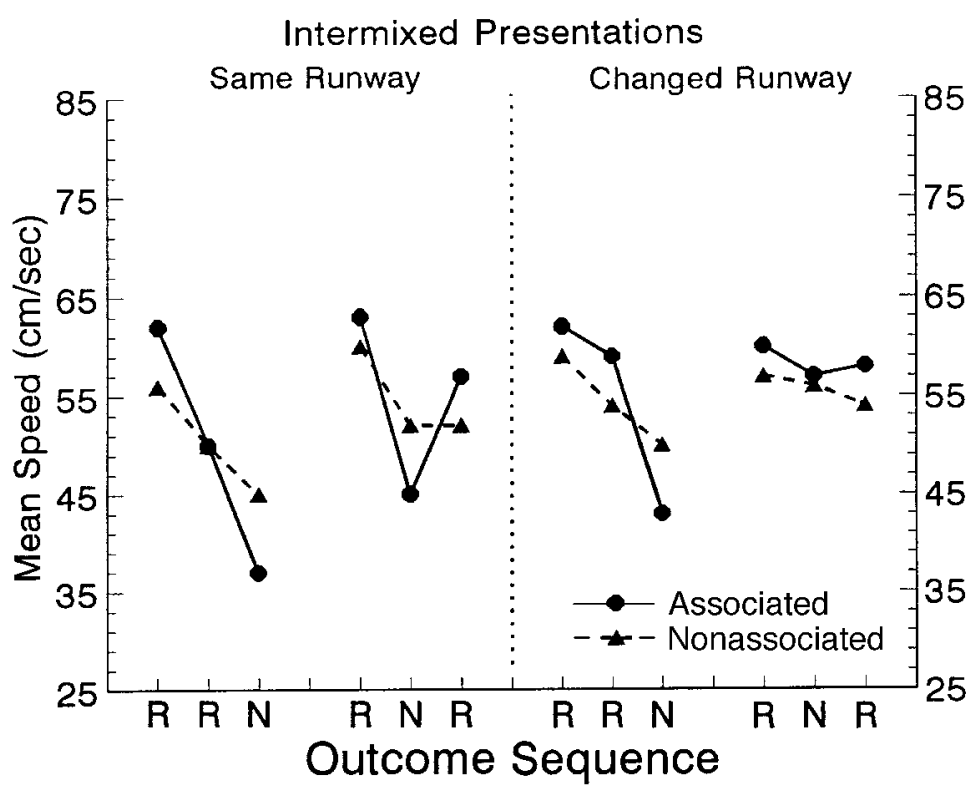

Figure 2. Mean running speed on each trial within each outcome sequence during intermixed forced-choice series presentations for each group when the runway on all trials remained the same (left panel) or was changed on Trials 2 and 3 from that on Trial 1 (right panel) in Phase 4 of Experiment 1. 
dicted by the intertrial association, sequential memory hypothesis. Contrary to that hypothesis, however, its serial pattern responding was enhanced within the RRN sequence. That is, both groups maintained similar speeds over all three trials within the RNR sequence, but the associatedrunways group reduced its speed, on the last trial within the RRN sequence, below that of the nonassociated-runways group.

\section{EXPERIMENT 2}

The results from Phase 4 in Experiment 1 suggest that the rats in the associated-runways group developed intertrial associations between the runway on the first trial and the runway and outcome on the next trial that were dominant over and overshadowed possible intratrial, ordinal position associations. This effect was evident primarily within the RNR sequence. Perhaps, if the first trial's runway had never become a reliable cue for the second trial's runway and outcome, the associated-runways group might have developed dominant intratrial associations to allow it to integrate RNR and RRN sequences. The second experiment tested this idea by assigning new rats to each group and exposing them, on the first trial, to a runway opposite that on the next two trials on half the series within each RRN and RNR sequence. A free-choice test phase followed forced-choice training to test integration based on intratrial associations in the associated-runways group and on intertrial associations in the nonassociated-runways group. A forced-choice test phase with RNN test sequences intermixed with RRN and RNR sequences was then conducted to determine the type of association each group used for its serial pattern responding. A final free-choice test phase was presented to determine whether the RNN sequence in the previous forced-choice phase improved choices of baited runways by the associated-runways group on the last two trials and by the nonassociated-runways group on the last trial.

If forced-choice training with RRN and RNR sequences created more dominant intratrial associations in the associated-runways group, its rats should choose the baited runways on both the second and the third free-choice trials during the first free-choice test phase. If intertrial associations remained dominant, then these rats, along with those in the nonassociated-runways group, should accurately select a baited runway only on the free-choice third trial. In the forced-choice phase with RNN test probes, intratrial associations in the associated-runways group would be evidenced by equally slow speeds on the last trial within the RNN and RRN sequences. Intertrial associations, however, would be revealed by faster speeds on the last trial within the RNN sequence than on that within the RRN sequence. Since the rats in the nonassociated-runways group could develop intertrial associations only between the outcomes of the second and the third trials, they were expected to run as fast on the final trial within the RNN probes as on that within the RNR sequence.

\section{Method}

\section{Subjects}

Twelve male Long-Evans hooded rats from the same subject pool and having had the same history as the rats in the previous experiment were used.

\section{Procedure}

Following preliminary shaping without being exposed to forcedand free-choice RRR sequence training as in Experiment 1, the rats were assigned to the associated- and nonassociated-runw ays groups and received five successive phases: Phase 1, separate-presenta tions training with RRN and RNR sequences for 48 sessions; Phase 2, intermixed presentations with these sequences for another 24 sessions; Phase 3, free-choice tests for 12 sessions with both runways open on each trial; Phase 4, intermixed presentations of RRN, RNR, and RNN (test probe) sequences for 32 sessions; Phase 5, free-choice tests as in Phase 3. Phases 1-3 were similar to those in Experiment 1, except that the runway on the first trial of the forced-choice series was not reliably associated with the runway on the next two trials.

During Phases 1 and 2, 3 rats in the associated-runways group were exposed to the RRN sequence whenever they were forced down the left runway on all three trials in a series (same-runway condition) or down the right runway on the first trial and then down the left runway on the next two trials in a series (changed-runway condition). They experienced the RNR sequence whenever they were forced down the right runway on every trial (same-runway condition) or down the left runway on the first trial and down the right runway on the next two trials (changed-runway condition). The remaining 3 rats in this group received the opposite runway pattern conditions (RRN, right-right-right or left-right-right; RNR, left-left-left or right-left-left). The rats in the nonassociated-runways group received the same- and the changed-runway conditions in the forcedchoice phases, but with each sequence being equally presented within each of the four runway variations (all left; all right; left-rightright; right-left-left). Each runway condition occurred in a random order without the same condition occurring on more than two series in a row within each forced-choice session.

In Phase 4, the rats received RRN, RNR, and RNN (test probe) sequences intermixed within each session for 32 sessions. Each sequence was presented twice within a session, once with the same runway on all three trials within the RRN and RNR sequences and on the first two trials within the RNN sequence and another time with the runway on the first trial opposite that on the next two trials within the RRN and RNR sequences and opposite that on the second trial within the RNN sequence. The runway on the third trial within RNN sequence probes, however, had to always be opposite that on the second trial. The runway on the third trial within the RRN and the RNN sequences was always the same for the rats in the associatedrunways group but could differ for the rats in the nonassociatedrunways group.

\section{Results and Discussion}

\section{Phases 1 and 2: Separate and Intermixed Presentations}

Figure 3 summarizes running speed data under each runway condition from the last 24 sessions within the separate-presentations phase (top graph) and from the 24 sessions within the intermixed-presentations phase (bottom graph). Similar effects occurred within each phase, as can be seen from each graph and was confirmed by an interaction among all factors in a four-way ANOVA (group $\times$ runway condition $\times$ sequence $\times$ trial) within each phase $[F \mathrm{~s}(2,20)=16.28$ and 17.68$]$. 

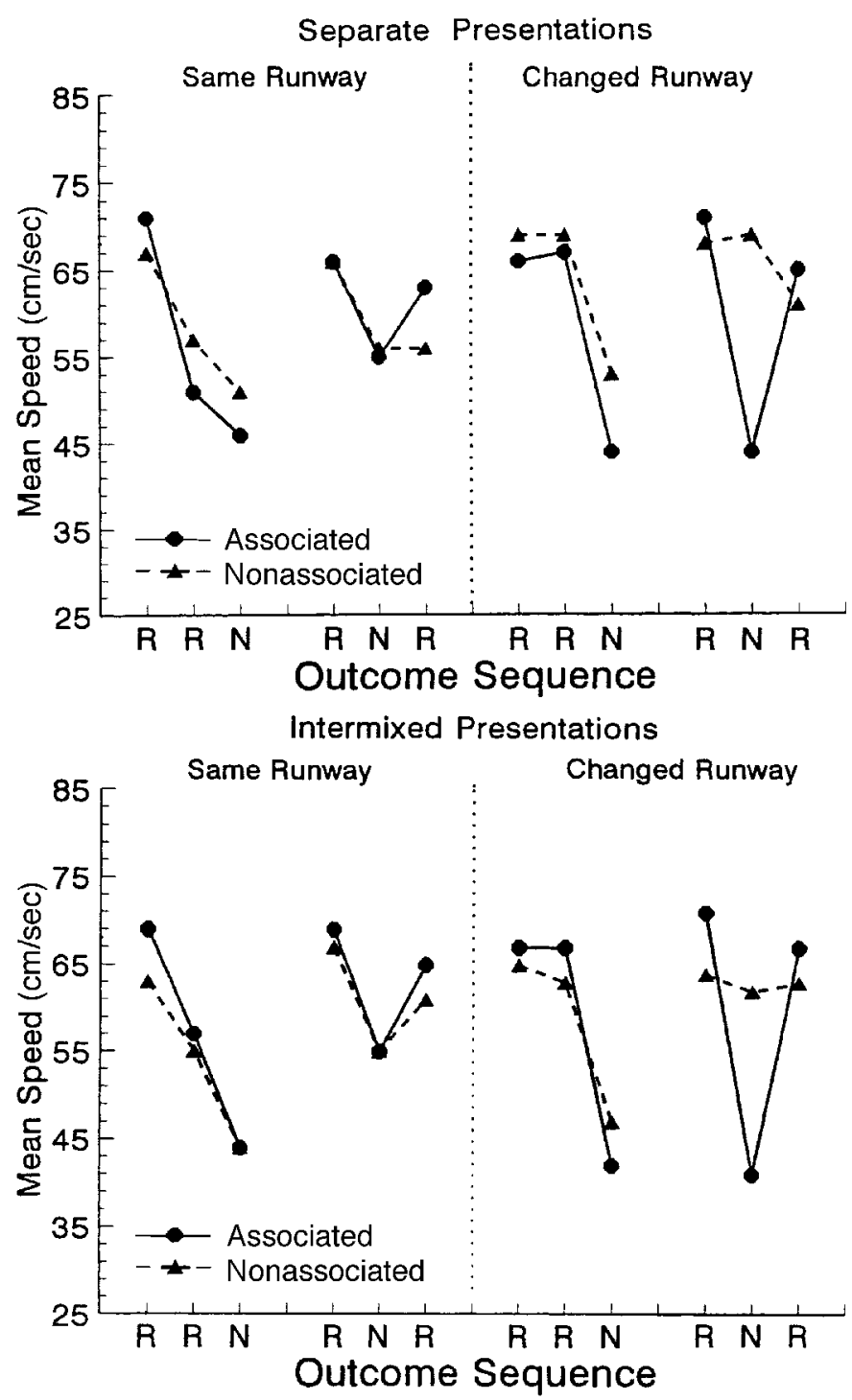

Figure 3. Mean running speed on each trial within each outcome sequence during separate and intermixed forced-choice series presentations for each group when the runway on all trials remained the same (left panels) or was changed on Trials 2 and 3 from that on Trial 1 (right panels) in Phase 1 (top graph) and Phase 2 (bottom graph) in Experiment 2.

Both groups showed similar serial running patterns, for the RRN sequence, by reducing their speeds on the second trial and reducing them further on the third trial under the same-runway condition but only doing so on the third trial under the changed-runway condition. Groups differed in their responding on the RNR sequence. The associated-runways group displayed a more consistent serial response pattern by reducing its speed only on the second trial under either runway condition within each phase. The nonassociated-runways group reduced its speed only on the second trial under the same-runway condition. Therefore, the latter group seemed unable to form as accurate an RNR list chunk during separate presentations as it had in Experiment 1. Moreover, the associated-runways group reduced its speed more on the second trial within the RNR sequence under the changed-runway condition than under the same-runway condition. Thus, changing the runway on the final two trials enhanced its serial pattern responding within the RNR sequence, rather than disrupting it.

\section{Phase 3: Free-Choice Test}

Figure 4 summarizes free choices in this phase (left panel) and in the final phase (right panel). As can be seen 


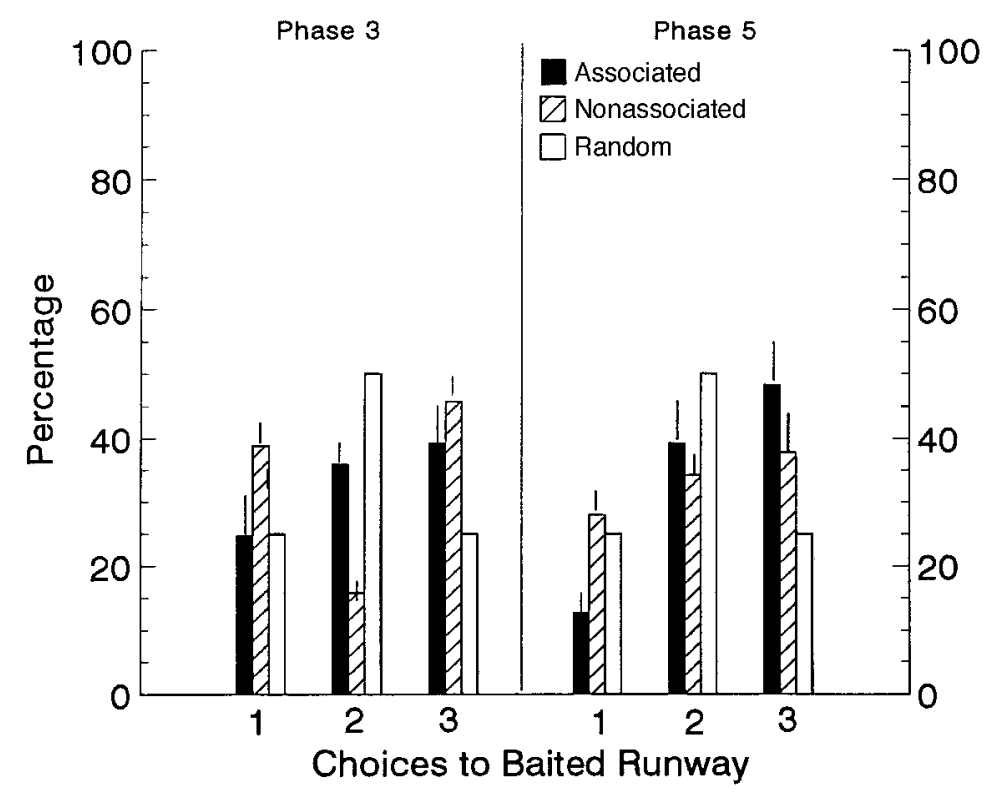

\begin{abstract}
Figure 4. Percentages of choices of a baited runway made on one, two, or all three trials during the free-choice testing on Phase 3 (left panel) and in Phase 5 (right panel) in Experiment 2 for each group, as compared with that which would have been made by random selections. Vertical lines at the top of mean data bars represent $+S E M$ s.
\end{abstract}

in the left panel, each group chose the baited runway on all three trials significantly more often than would be expected by chance $\left[F_{\mathrm{s}}(1,5)=43.01\right.$ and 271.69$]$ and, on two trials, significantly less often than would be expected by chance $[F \mathrm{~s}(1,5)=84.11$ and 215.84$]$, but only the nonassociated group chose a baited runway only on the first trial more often than would be expected by chance $[F(1,5)=30.80]$. The associated-runways group selected a baited runway on three trials significantly more often than on only one trial $[F(1,5)=16.25]$, but not significantly more often than on only two trials. A further examination of choice patterns revealed that the rats in the associated-runways group almost always chose a baited runway on the third trial after choosing one on the second trial. When choosing a baited runway on only two trials, however, they usually did so by choosing a nonbaited runway on the second trial and then choosing the same but now baited runway on the final trial. Thus, these rats were able to determine which runway would be baited on the third trial by remembering the outcome and the runway chosen on the second trial. The nonassociatedrunways group displayed a different pattern by selecting a baited runway on three trials significantly more often than on only two trials $[F(1,5)=109.96]$, but not more often than on only the first trial. The nonassociatedrunways group selected a baited runway more often only once or on all three trials $\left[F_{\mathrm{s}}(1,10)=21.01\right.$ and 6.66], but less often on two trials $[F(1,10)=52.86]$, than did the associated-runways group. Thus, contrary to expectations, the rats in the nonassociated-runways group were unable to accurately determine which runway was baited on the final free-choice trial from the outcome of their selected runway on the second free-choice trial.

\section{Phase 4: Intermixed Presentations of RRN, RNR, and RNN Sequences}

Figure 5 shows the mean running speeds on each trial within each sequence, collapsed over runway condition for each group over the last 16 sessions. These data were analyzed with a four-way ANOVA similar to that in Phases 1 and 2. Runway condition continued to affect running speeds within the RRN sequence in same manner as in Phase 2. As can be seen in Figure 5 and was confirmed by a significant group $\times$ sequence $\times$ trial interaction $[F(4,40)=14.76]$, both groups reduced their speeds on the last trial within the RRN sequence, but only the associated-runways group consistently ran more slowly on the second than on the first trial within either the RNR or the RNN sequence and, more important, increased its speed on the last trial within either of these sequences, as predicted by the intertrial association hypothesis. This increase was significantly greater within the RNR sequence than within the RNN sequence, however, suggesting that intratrial associations on the third trial also contributed to responding on that trial.

\section{Phase 5: Free-Choice Test}

As can be seen in the right panel of Figure 4, the percentage of times the rats chose a baited arm on only the first trial declined from that in Phase 3 either to signifi- 
cantly below chance $[F(1,5)=51.92]$ in the associatedrunways group or to chance in the nonassociated-runways group. Both groups selected a baited arm on two trials about as often as they did on every trial which they chose significantly more often than only on the first trial $[F(1,5)=$ 16.04]. At first glance, these effects conform to the intertrial association hypothesis. However, the rats may have learned, from their forced-choice exposures to an RNN sequence, not to switch runways after encountering a nonbaited runway on the second trial.

\section{EXPERIMENT 3}

The results from Experiment 2 suggest that intertrial associations between the second and the third trial outcomes were dominant over possible intratrial associations between the third trial's runway and its outcome, for the associated-runways group. This conclusion conflicts with those offered by Burns et al. (1999), who found intratrial associations between runway and outcome on the third trial in $\mathrm{ZNN}$ and $\mathrm{XNY}$ sequences to be dominant over intertrial associations between the type of food on the first trial and the outcome on the last trial. Perhaps the presence and absence of food as a signal for outcome on the next trial in our experiments created stronger (and, hence, dominant) intertrial associations than those created from type of food on the first trial and outcome on the third trial in Burns et al. (1999). Consequently, we conducted a final experiment, to determine whether more remote intertrial associations between the first and the third trial outcomes would also be dominant over intratrial associations based on runway cues at each ordinally tagged trial.

New rats in the associated-runways and nonassociatedrunways groups were initially trained on NRR and RRN se- quences under separate (Phase 1) and intermixed (Phase 2) presentations. Following this training, the rats received a third phase with RRR and NRN sequences intermixed with the original NRR and RRN sequences. In this phase, however, the rats in the nonassociated group also had runways consistently paired with each outcome on the first and the third trials, to determine whether they would learn the predictive relevance of previous irrelevant runway cues. The rats in the associated-runways group should run more slowly on a nonbaited runway than on a baited runway on the third trial, regardless of the runway on the first trial, if they developed dominant intratrial associations. However, if they formed dominant or only intertrial associations between first and third trial outcomes, they should run more slowly down either runway on the third trial following a baited runway than following an unbaited runway on the first trial, as might the rats in the nonassociated-runways group. A fourth free-choice phase was also conducted to determine whether prior exposure to all possible runwaycued sequences would promote selection of a baited runway on every trial.

\section{Method}

\section{Subjects}

Twelve male Long-Evans hooded rats from the same subject pool, having the same history as those in the previous two experiments, served in this experiment. Two rats were dropped from this experiment, one owing to illness and the other owing to failure to run in the apparatus during initial training, leaving 5 rats in each group.

\section{Procedure}

Following the same initial training procedures as those in Experiment 2 , the rats were assigned to the associated-r unways and nonassociated-runways groups and were exposed to RRN and NRR sequences for 72 sessions under the same-runway condition. These

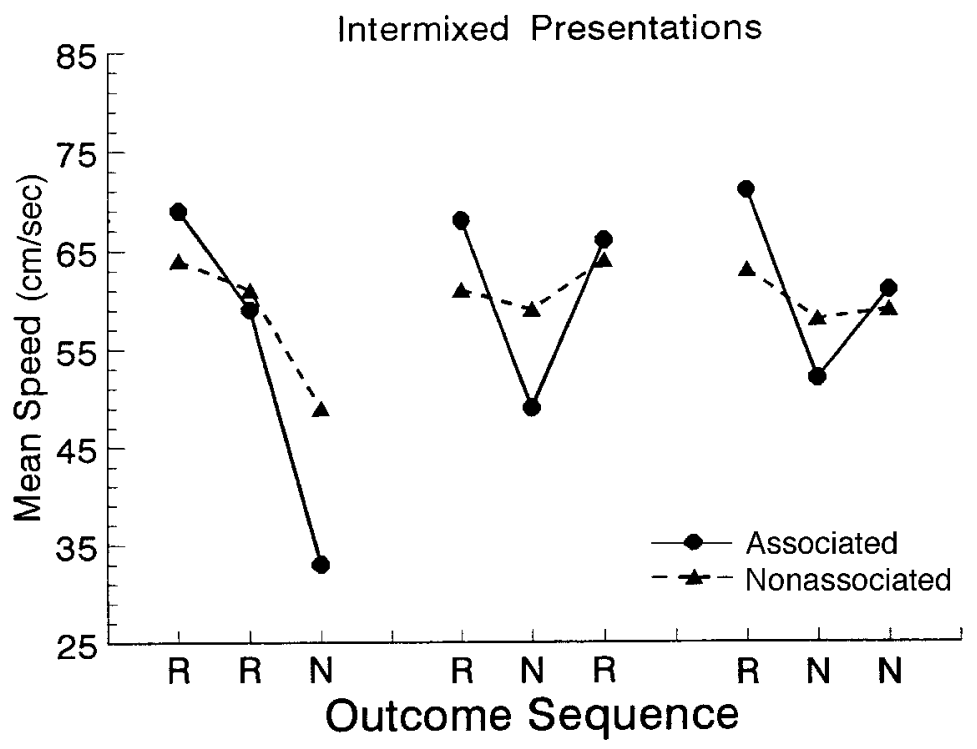

Figure 5. Mean running speed on each previously acquired outcome sequence, RRN and RNR, and an added test outcome sequence, RNN, over the last 16 sessions for each group in Phase 4 of Experiment 2. 
sequences were presented separately over the first 48 sessions (Phase 1) and then were intermixed within each of the remaining 24 sessions (Phase 2). Phase 3, consisting of 32 sessions, contained new RRR and NRN sequences intermixed with the original RRN and NRR sequences. Each of these forced-choice test sessions contained six series in which each of the original sequences, RRN and NRR, occurred once and each of the new sequences, RRR and NRN, occurred twice. As was noted, the rats in the nonassociated-runways group had these sequences paired with runways in the same way as those in the associated-runways group. For 3 animals in the nonassociatedrunways group, only the left runway was baited on the first trial, and the right runway was baited only on the third trial. The oppositerunway-outcome pairings occurred for the other 2 animals. The only other change in this phase was that the runway on the second trial was changed from that on the first trial on half the series for each sequence so as to vary equally within each session for the test sequences and over sessions for the original sequences. Phase 4 consisted of 12 sessions of free-choice series, as in Experiment 2, except that the outcomes on the first and the third trials remained associated with specific runways, as established in Phase 3.

\section{Results}

\section{Phases 1 and 2: RRN and NRR Sequences}

Figure 6 shows mean speeds on the final block of 24 sessions during the separate-presentations phase (left panel) and the intermixed-presentations phase (right panel). The left panel shows that both groups ran more slowly on each sequence's $N$ trial during Phase 1 , with the associated-runways group developing slower speeds, as was confirmed by a significant group $\times$ sequence interaction $[F(2,16)=9.18]$. As can be seen in the right panel and was confirmed by a significant three-way interaction $[F(2,16)=30.69]$, both groups similarly reduced their speeds on the last trial within the RRN sequence, but only the associated-runways group continued to run significantly more slowly on the first trial within the NRR sequence.

\section{Phase 3: Intermixed Presentations of RRR, NRN, RRN, and NRR Sequences}

Figure 7 shows mean running speeds within each sequence over the first (left panel) and last (right panel) 16 sessions of this phase for each group. Separate three-way ANOVAs were conducted on RRR and NRN sequences and on RRN and NRR sequences for each block of 16 sessions. The first analysis (RRR vs. NRN) revealed that the associated-runways group developed more accurate serial pattern responding than did the former nonassociatedrunways group on the new sequences during the first 16 sessions, as can be seen in the left panel and was confirmed by an interaction among all three factors $[F(2,16)=$ 5.77]. The associated-runways group did not significantly vary its speeds over trials within the RRR sequence but ran more slowly on both $\mathrm{N}$ trials within the NRN sequence. The former nonassociated-runways group slightly but significantly reduced its speed on the third trial within the RRR sequence and ran significantly more slowly only on the third trial within the NRN sequence. On the final 16 sessions (right panel), the former nonassociatedrunways group developed running patterns similar to those in the associated-runways group by also running more slowly on the first and third trials than on the second trial within the NRN sequence. Although the former nonassociated-runways group apparently continued to

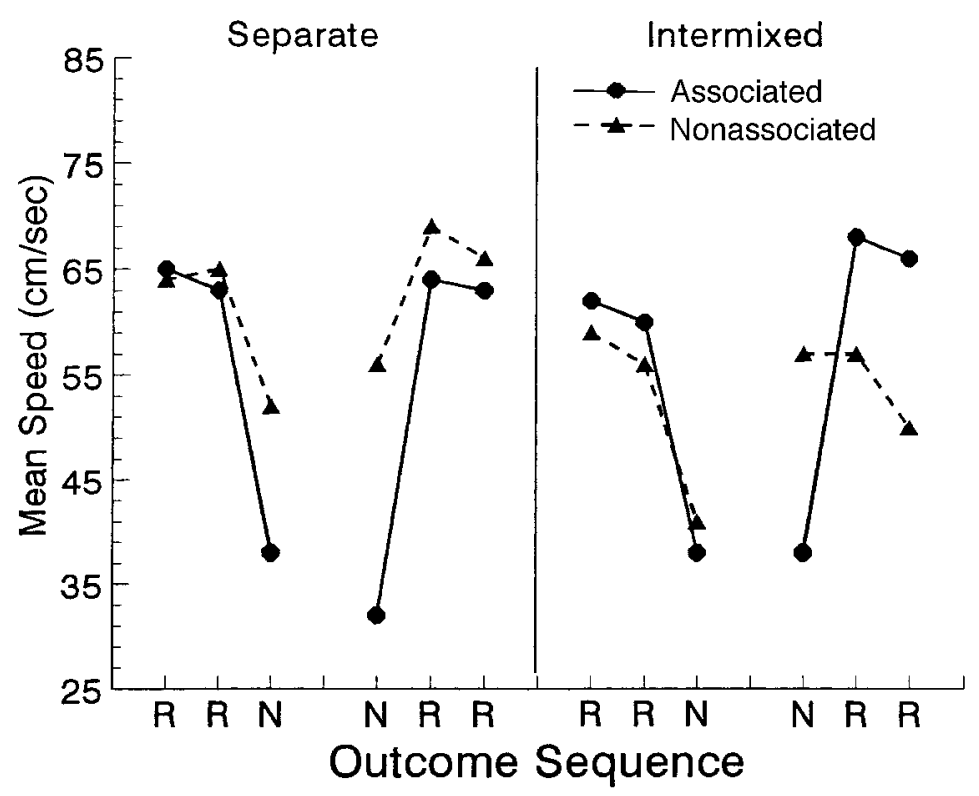

Figure 6. Mean running speed on each trial within each outcome sequence, NRR and RRN, during separate (Phase 1) and intermixed (Phase 2) forcedchoice series presentations for each group in Experiment 3. 


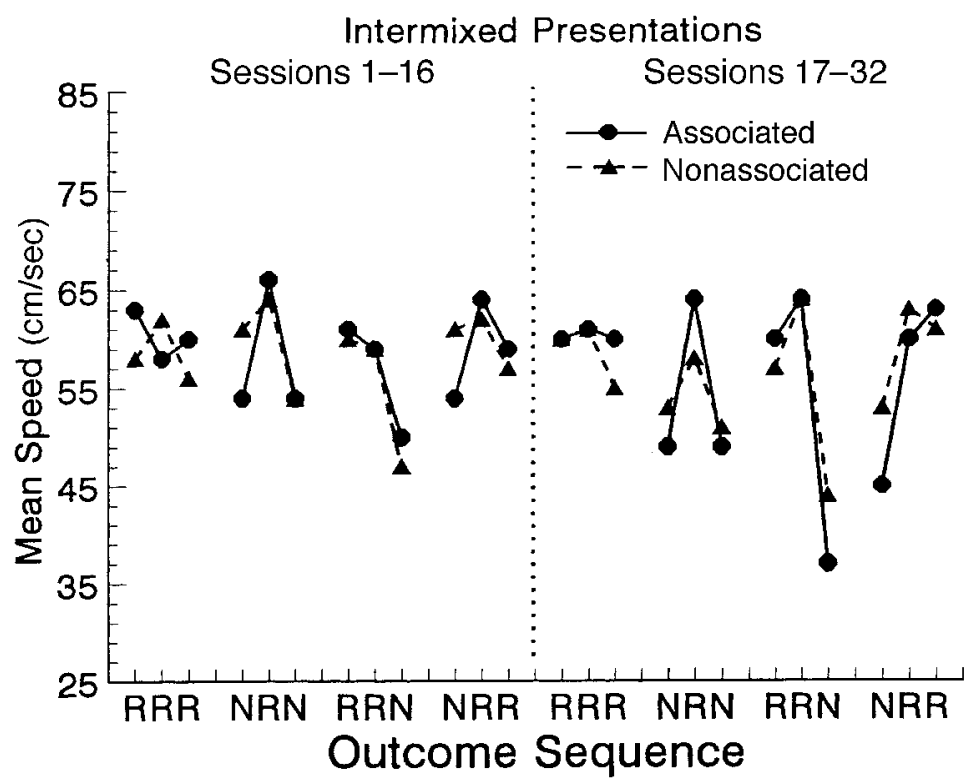

\begin{abstract}
Figure 7. Mean running speed on each trial within the added test outcome sequences, RRR and NRN, and the previously acquired outcome sequences, RRN and NRR, for each group over the first and last 16 sessions in the forcedchoice series test (Phase 3) in Experiment 3.
\end{abstract}

reduce its speed on the last trial within the RRR sequence, neither a three-way interaction $[F(2,16)=3.17, p=.07]$ nor individual comparisons confirmed this observation. Although both groups displayed similar slower speeds on $\mathrm{N}$ trials within the original sequences (RRN and NRR), especially over the last 16 sessions, the associatedrunways group ran more slowly on the $\mathrm{N}$ trial within the NRR sequence over the first 16 sessions and within the RRN and NRR sequence over the second 16 sessions than did the former nonassociated-runways group. Significant individual comparisons over the first and second blocks of 16 sessions and a significant three-way interaction $[F(2,16)=4.44]$ on the second block of 16 sessions confirmed these observations.

These findings suggest that the rats in the associatedrunways group developed dominant intratrial associations on the third trial, as well as on the first trial, in the originally acquired sequences, which allowed them to disregard the first trial's outcome within either test sequence. The nonassociated-runways group developed intertrial associations between the first and the third trial outcomes during original training, however, which affected its performance on the test sequences. That is, this group displayed reliable intratrial associations between runway cues and outcomes on the first and third trials similar to those of the associated-runways group within the last 16 sessions but not within the first 16 sessions.

\section{Phase 3: Free-Choice Tests}

Figure 8 shows the percentage of choices in which each group selected a baited runway on only one trial (the second), on only two trials, or on all three free-choice trials.
As can be seen in this figure and was statistically confirmed, the associated-runways group chose a baited runway on every trial more often than on either only two trials or only one trial $\left[F_{\mathrm{s}}(1,4)=19.97\right.$ and 85.02$]$. Four rats in this group selected a baited runway on all trials more than $70 \%$ of the time, and 1 rat $51 \%$ of the time. The nonassociated-runways group also chose a baited runway on all three trials significantly more often than on only one trial $[F(1,4)=55.81]$, but not significantly more often than on only two trials. Four rats in that group chose a baited runway on every trial between $54 \%$ and $60 \%$ of the time, and 1 rat $27 \%$ of the time. As is also evident in this figure and was confirmed by significant differences between groups $\left[F_{\mathrm{S}}(1,8)=7.54\right.$ and 5.98], the associatedrunways group chose a baited runway on all three trials more often and on two trials less often than did the nonassociated-runways group. Both groups seldom chose a baited runway only once, however. These results suggest that the rats in the associated-runways group were, at least, better able to transfer specific forced-choice runway patterns for RRR sequences in Phase 3 to the free-choice phase than were the rats in the nonassociated-runways group.

\section{GENERAL DISCUSSION}

The results from the first two experiments in this study replicate serial pattern responding in Cohen, Westlake, and Pepin (2001). That is, serial pattern responding within RRN and RNR sequences, separately presented over sessions, was better maintained during intermixed presentations within sessions when each sequence was associated 


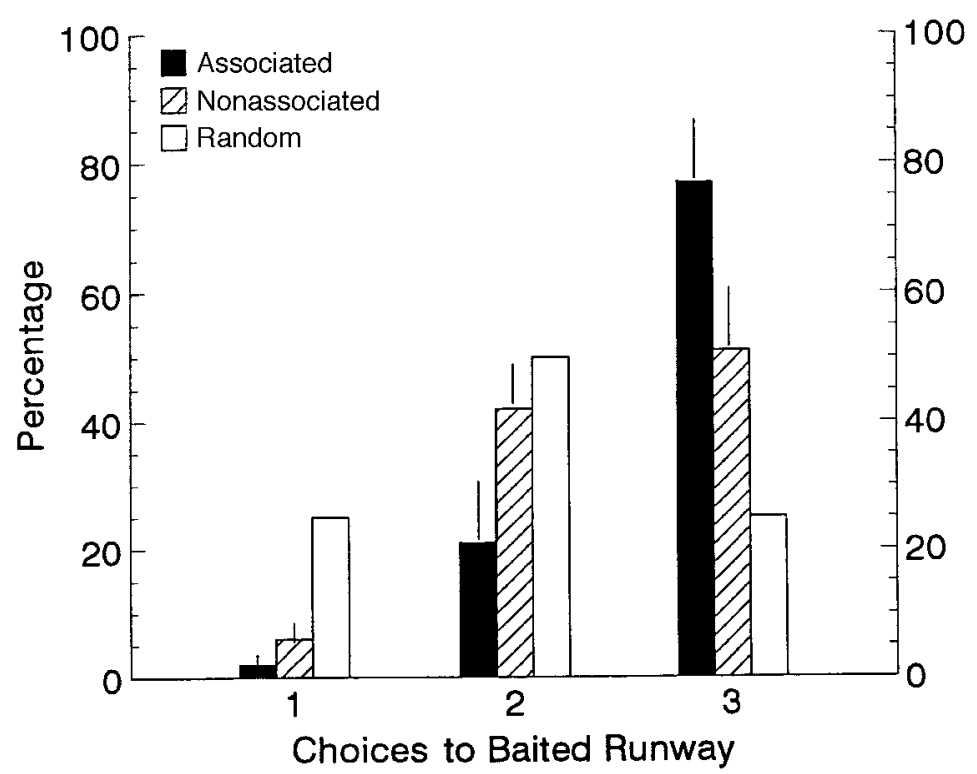

Figure 8. Percentage of choices of a baited runway made on one, two, or all three trials during free-choice test (Phase 4) for each group, as compared with that which would have been made by random selections in Experiment 3. Vertical lines at the top of mean data bars represent $+S E M s$.

with a specific, distinctive runway. The present study extended these findings, however, by determining which of two serial pattern representations was dominant for rats in the associated-runways group. In the first two experiments, intertrial runway-outcome associations between the last two trials were dominant over intratrial runwayoutcome associations within each trial. In the third experiment, in which more remote intertrial associations between the first and the third trials could occur along with intratrial associations, the latter were dominant.

Burns, Kinney, and Criddle (2000) found that, although close intertrial associations affect ease of acquiring serial pattern behavior, intratrial associations based on ordinal trial positions were dominant on postacquisition test sequences. In their Experiment 1, the rats exposed to a consistent change in food type over the first two trials preceding a final nonreinforced trial reduced that final trial's running speed earlier in training than did the rats exposed to random variations in food type, a result comparable to earlier findings (Capaldi, Alptekin, Miller, \& Birmingham, 1997). When exposed to an NNN test sequence, however, both groups continued to run more slowly on the third trial than on the first two trials, as predicted by the ordinal trial position hypothesis. In their Experiment 2, a second nonrewarded trial always preceded a third trial with an amount of food twice that on the first trial. As predicted by the ordinal trial position hypothesis and contrary to the intertrial association notion, the rats continued running slower on the second trial within the postacquisition NNN sequence.

The less accurate serial pattern responding occurring under same- than under changed-runway condition in Experiment 2 requires some comment. The rats reduced running speeds on the second trial within both sequences under the same-runway condition. Under the changedrunway conditions, however, they maintained them over the first two trials within the RRN. At least for the nonassociated-runways group, these effects may be attributed to differences in the predictive validity of a reinforced trial as a signal for the outcome on the next trial. When all possible two-trial subsequences beginning with an $\mathrm{R}$ trial are examined, $R N$ subsequences occurred twice as often without any runway change $(R N \mathrm{R}$ and $\mathrm{R} R N$ during the same-runway condition) than with a change ( $R N R$ during the changed-runway condition) but $R R$ subsequences occurred as often under either runway variation ( $R R \mathrm{~N}$ during the same-runway condition and $R R \mathrm{~N}$ during the changed-runway condition). Consequently, the rats in the nonassociated-runways group could learn that an unbaited runway more likely followed a baited one when the runway remained the same than when it was changed.

Runway context manipulations have produced comparable effects in outcome expectancies between groups in which different sequences were also not associated with specific runways (Capaldi, Birmingham, \& Miller, 1999; Capaldi \& Miller, 2001). In those studies, some experimental rats experienced a runway change on the last trial of a series only when it ended with an $\mathrm{R}$ trial, rather than with an $\mathrm{N}$ trial, where either trial always followed a previous $\mathrm{N}$ trial. Other, control rats experienced the same change in runway on either type of trial (Capaldi et al., 1999, Experiment 2) or only on an R trial following a longer sequence of $\mathrm{N}$ trials (Capaldi \& Miller, 2001, Experiment 1 ). Only the experimental rats were able to accurately predict the outcome of the target trial in a sequence. Thus, a change in runways between trials was a 
more relevant predictor of trial outcome than was the previous trial's outcome in the experimental group.

Although the associated-runways group also reduced its speed on the second trial within each sequence under the same-runway condition, it also did so on the second trial within the RNR sequence under the changed-runway condition, contrary to the preceding explanation. The rats in this group may have partitioned each series into two successive chunks, a single R trial followed by a two-trial RN or NR chunk, each of the latter associated with a specific runway. Changing runways between the first trial and the next two trials in some series may have provided an additional phasing cue, allowing these rats to more easily differentiate NR from RN subsequences than when this phasing cue was absent under the same-runway condition. Therefore, in terms of Capaldi's (1992) hierarchical chunking model, list chunking for some sequences may require consistent phrasing or grouping cues over all series presentations. This speculation corresponds with findings that rats are better able to form a list chunk from two different sequences, RNN and RNR, when they are consistently separated by temporal or contextual phrasing cues (Haggbloom, 1993; Haggbloom, Birmingham, \& Scranton, 1992).

Unlike the results from the free-choice tests in Cohen, Westlake, and Pepin (2001), the rats in the associatedrunways group were better able to determine which runway would be baited on the last free-choice trial than were the rats in the nonassociated-runways group in Experiments 1 and 2 . The fact that the former did not predominantly select a baited runway on every trial prevents us from concluding that they had integrated the runwaycued sequences into a higher order, RRR super chunk. Rather, they more likely used the runway and outcome on the second trial as a signal for which runway would be baited on the last trial, a conditional intertrial association. The rats in the nonassociated-runways group could not accomplish this until they had been exposed to an RNN sequence in Experiment 2, which probably reduced the tendency to switch runways after encountering a nonbaited one. Only after experiencing all possible runway sequences (Experiment 3, Phase 3) did the associatedrunways group predominantly choose a baited arm on all free-choice trials and did the nonassociated-runways group choose it on the third trial. The rats may have learned which forced-choice sequences of runway presentations produced more rewarded trials and tried to reproduce them during free-choice series. That the associatedrunways group was more successful than the nonassociatedrunways group might be owing to their greater exposure to intratrial associations between runways and outcomes.

We note that the rats in the associated-runways group usually chose a baited runway on the first trial and then chose the other runway on the next two trials in Experiment 3 . They seldom chose the same runway on the first two trials, and then chose the other runway on the last trial. Perhaps changing the runway after the first rewarded trial provided a more salient grouping cue within the forced-choice RRR sequences, which these animals could better reproduce during free-choice conditions. This explanation is similar to one advanced by Fountain, Benson, and Wallace (2000). In their study, rats obtained reinforcing brain stimulation for pressing levers in a clockwise manner with a counterclockwise choice of a lever after every third correct lever choice (e.g., 123/ 234/345/456/567/781/812, where / refers to a series boundary). Acquisition of this difficult pattern was facilitated the more often interseries intervals (temporal phrasing cues) that differed from within-series lever choice intervals occurred. More important, errors on the first lever choice within each series were more likely on series not separated by a distinct phrasing cue. In Experiment 3 of the present study, the rats in the nonassociated-runways group may have been unable to consistently choose a baited runway on all free-choice trials because they had not yet learned which runway was baited on the first trial. Thus, the opposite runway on the second trial had not become a salient phrasing cue for this group.

The idea that a more complete or adequate understanding of our universe requires integration of incomplete or even disparate information defines our notion of scientific discovery. A fundamental question remains whether this human ability is a general characteristic in animals. Rats, as central place foragers, would benefit not only from recognizing consistent temporal patterns of food availability within different areas of their environment, but also from integrating them for more efficient exploitation of their resources. To do this, however, would require that they form global representations, rather than simpler associative representations, of these patterns. Although we have uncovered some conditions that favor the former over the latter, it remains to be seen whether these and other conditions promote such integration.

\section{REFERENCES}

Bower, G. H., \& Springston, F. (1970). Pause as recoding points in letter series. Journal of Experimental Psychology, 83, 421-430.

Bower, G. H. \& Winzenz, D. (1969). Group structure, coding, and memory for digit series. Journal of Experimental Psychology, 80, 12-22.

Burns, R. A., Dunkman, J. A., JR., \& Detloff, S. L. (1999). Ordinal position in the serial learning of rats. Animal Learning \& Behavior, 27, 272-279.

Burns, R. A., Kinney, B. A., \& Criddle, C. R. (2000). Position cues and reward memories as compatible components of serial learning. Learning \& Motivation, 31, 236-250.

Burns, R. A., Wiley, L. P., \& Payne, T. L. (1986). Temporal cuing of runs in series of reward events reduces interevent anticipation. Animal Learning \& Behavior, 14, 190-196.

CAPAldi, E. J. (1992). Levels of organized behavior. In W. K. Honig \& G. Fetterman (Eds.), Cognitive aspects of stimulus control (pp. 385404). Hillsdale, NJ: Erlbaum.

Capaldi, E. J., Alptekin, S., Miller, D. J., \& Birmingham, K. M. (1997). Is discriminative responding in reward outcome serial learning mediated by item memories or position cues? Learning \& Motivation, 28, 153-169.

Capaldi, E. J., Birmingham, K. M., \& Miller, R. M. (1999). Forming chunks in instrumental learning: The role of overshadowing. Animal Learning \& Behavior, 27, 221-228.

Capaldi, E. J., \& Miller, R. M. (2001). Molar vs. molecular approaches to reward schedule and serial learning phenomena. Learning \& Motivation, 32, 22-35. 
Capaldi, E. J., Miller, D. J., Alptekin, S., \& Barry, K. (1990). Organized responding in instrumental learning: Chunks and superchunks. Learning \& Motivation, 21, 415-433.

Capaldi, E. J., \& Molina, P. (1979). Element discriminability as a determinant of serial-pattern learning. Animal Learning \& Behavior, 7, 318-322.

Capaldi, E. J., Nawrocki, T. M., \& Verry, D. R. (1983). The nature of anticipation: An inter- and intraevent process. Animal Learning \& Behavior, 11, 193-198.

CAPAldi, E. J., \& VerRy, D. R. (1981). Serial order anticipation learning in rats: Memory for multiple hedonic events and their order. Animal Learning \& Behavior, 9, 441-453.

CApaldi, E. J., Verry, D. R., Nawrocki, T. M., \& Miller, D. J. (1984). Serial learning, interitem associations, phrasing cues, interference, overshadowing, chunking, memory, and extinction. Animal Learning \& Behavior, 12, 7-20.

Cohen, J. S., Drummond, C., \& Terrelonge,N. (2001). Value transfer in simultaneous object discriminations by rats. Animal Learning \& Behavior, 29, 326-335.

Cohen, J. S., Westlake, K., \& Pepin, M. (2001). Higher-order chunking in rats' serial pattern learning in the T-maze. Learning \& Motivation, 32, 409-433.

Fountain, S. B., Benson, A. M., \& Wallace, D. G. (2000). Number, but not rhythmicity, of temporal cues determines phrasing effects in rat serial-pattern learning. Learning \& Motivation, 31, 301-322.

Fountain, S. B., Henne, D. R., \& Hulse, S. H. (1984). Phrasing cues and hierarchical organization in serial pattern learning in rats. Journal of Experimental Psychology: Animal Behavior Processes, 10, 30-45.

HagGbLoom, S. J. (1993). Positive transfer across group cue modalities in rat serial learning. Learning \& Motivation, 24, 266-281.

Haggbloom, S. J., Birmingham, K. M., \& Scranton, D. B. (1992). Hierarchical organization of series chunks and list chunks. Learning \& Motivation, 23, 183-199.

Hulse, S. H. (1978). Cognitive structure and serial pattern learning by animals. In S. H. Hulse, H. Fowler, \& W. K. Honig (Eds.), Cognitive processes in animal behavior (pp. 311-340). Hillsdale, NJ: Erlbaum.

(Manuscript received November 13, 2001; revision accepted for publication May 20, 2002.) 